\title{
High-energy Behavior of the Scattering Amplitude for a Dirac Operator
}

By

\author{
Hiroshi T. Iто*
}

\begin{abstract}
We study the high-energy behavior of the scattering amplitude and the total scattering cross section for a Dirac operator with a $4 \times 4$ matrix-valued potential. Moreover, in the electro-magnetic case, it is shown that the electric potential and the magnetic field can be reconstructed from the high-energy behavior of the scattering amplitude. The study of the high-energy behavior of the resolvent estimates is crucial for our proof.
\end{abstract}

\section{§ 1. Introduction}

The aim of this paper is to study the high-energy behavior of the scattering amplitude and the total scattering cross section for a Dirac operator. Moreover, in the electro-magnetic case, we show that the electric potential and the magnetic field (not the magnetic potential) can be reconstructed from the high-energy behavior of the scattering amplitude.

We define $4 \times 4$ matrices

$$
\alpha_{j}=\left(\begin{array}{cc}
0 & \sigma_{j} \\
\sigma_{j} & 0
\end{array}\right), \quad 1 \leq j \leq 3, \quad \beta=\left(\begin{array}{cc}
I_{2} & 0 \\
0 & -I_{2}
\end{array}\right),
$$

where $I_{n}$ is the $n \times n$ unit matrix and

$$
\sigma_{1}=\left(\begin{array}{ll}
0 & 1 \\
1 & 0
\end{array}\right), \quad \sigma_{2}=\left(\begin{array}{cc}
0 & -i \\
i & 0
\end{array}\right), \quad \sigma_{3}=\left(\begin{array}{cc}
1 & 0 \\
0 & -1
\end{array}\right)
$$

are the Pauli matrices. Then the matrices $\alpha$, and $\beta$ satisfy the relation:

$$
\alpha_{j} \alpha_{k}+\alpha_{k} \alpha_{j}=2 \delta_{j k}, \quad \alpha_{j} \beta+\beta \alpha_{\jmath}=0 \quad(1 \leq j, k \leq 3)
$$

Communicated by T. Kawai, May 25, 1995.

1991 Mathematics Subject Classification(s): 81U10, 81U20, 35P25.

* Department of Mathematics, Kyoto University, Kyoto 606-01, Japan. 
where $\delta_{j k}$ denotes the Kronecker symbol. With the matrices the free Dirac operator can be written as follows:

$$
H_{0}=c \alpha \cdot D+\beta m c^{2}=c \sum_{j=1}^{3} \alpha, D_{j}+\beta m c^{2} \text { in } \mathscr{H}=L^{2}\left(\boldsymbol{R}^{3} ; C^{4}\right),
$$

where $c>0$ is the velocity of light, $m>0$ is the rest mass of the particle, and $\alpha=\left(\alpha_{1}, \alpha_{2}, \alpha_{3}\right), D=\left(D_{1}, D_{2}, D_{3}\right), D_{j}=-i \partial / \partial x_{j}$. The free Dirac operator $H_{0}$ is a self-adjoint operator with domain $D\left(H_{0}\right)=H^{1}\left(\boldsymbol{R}^{3} ; \boldsymbol{C}^{4}\right)$, the Sobolev space of order 1 , and has purely absolutely continuous spectrum:

$$
\sigma\left(H_{0}\right)=\sigma_{a c}\left(H_{0}\right)=\left(-\infty,-m c^{2}\right] \cup\left[m c^{2}, \infty\right)
$$

The symbol $h(p):=c \alpha \cdot p+\beta m c^{2}, p \in \boldsymbol{R}^{3}$, of $H_{0}$ has two eigenvalues $\pm \lambda(p):=$ $\pm \sqrt{c^{2}|p|^{2}+m^{2} c^{4}}$, and each eigenspace $X^{ \pm}(p)$ is a two-dimensional subspace of $C^{4}$. Each element of $X^{ \pm}(p)$ describes an internal (spin) state of the free particle with momentum $p$.

When a magnetic potential $A(x)=\left(A_{1}(x), A_{2}(x), A_{3}(x)\right)$ and an electric potential $\phi(x)$ exist, the Dirac operator has the form:

$$
\begin{aligned}
H & =c \alpha \cdot(D-A)+\beta m c^{2}+\phi \\
& =H_{0}+V_{A \phi},
\end{aligned}
$$

where

$$
V_{A \phi}=-c \alpha \cdot A+\phi
$$

(The factor $e / c$ in front of $A$ is omitted for simplicity, where $e$ is charge of the particle.) We assume the following.

Assumption 1. There exist a real $\rho>2$ and an integer $d \geq 2$ such that each $v=A_{1}, A_{2}, A_{3}, \phi$ is in $C^{d}\left(\boldsymbol{R}^{3} ; \boldsymbol{R}\right)$ and obeys

$$
\left|\partial_{x}^{\gamma} v(x)\right| \leq C(1+|x|)^{-\rho}, \quad|\gamma| \leq d
$$

for some $C>0$.

In Sect. 2 we will define the scattering amplitude $F(E, \theta, \omega)$, which is a linear map from $X^{ \pm}(\nu(E) \omega)$ to $X^{ \pm}(\nu(E) \theta)$, for energy $E$ with $\pm E>m c^{2}$, initial direction $\omega \in S^{2}$ and scattering direction $\theta \in S^{2}$, where $S^{2}$ is the unit sphere in $\boldsymbol{R}^{3}$ and

$$
\nu(E)=c^{-1}\left(E^{2}-m^{2} c^{4}\right)^{1 / 2} .
$$


For a normalized initial state $u \in X^{ \pm}(\nu(E) \omega),|u|=1$, the total scattering cross section is defined by

$$
\sigma(E, \omega, u)=\int_{s^{2}}|F(E, \theta, \omega) u|^{2} d \theta
$$

It will be shown in Sect. 2 that the scattering amplitude is well-defined for each $(E, \theta, \omega)$ if $\rho>3$ and the total scattering cross section is well-defined for each $(E, \omega)$ if $\rho>2$.

To state our results on the high-energy asymptotics of $F(E, \theta, \omega)$ and $\sigma(E, \omega, u)$, we need some notations: For each $\omega \in S^{2}$ let $\Pi_{\omega}$ denote a plane orthogonal to $\omega, \Pi_{\omega}:=\left\{\eta \in \boldsymbol{R}^{3} \mid \eta \cdot \omega=0\right\}$, and define

$$
v_{\omega}(x):=\phi(x)-\omega \cdot c A(x),
$$

and

$$
m^{ \pm}(\omega):=\frac{1}{2 \pi c i} \int_{\Pi_{\omega}}\left\{\exp \left(-\frac{i}{c} \int_{-\infty}^{\infty} v_{ \pm \omega}(s \omega+\eta) d s\right)-1\right\} d \eta
$$

where the integral with respect to $\eta$ is absolutely convergent if $\rho>3$. Let $\boldsymbol{B}(X, Y)$ denote the space of bounded operators from a Banach space $X$ to a Banach space $Y$ and set $\boldsymbol{B}(X):=\boldsymbol{B}(X, X)$.

Theorem 1.1. Suppose Assumption 1 with $\rho>3$ and fix $\omega \in S^{2}$.

(i) Let $K$ be an arbitrary compact set of $S^{2} \backslash\{\omega\}$. Then

$$
\sup _{\theta \in K}\|F(E, \theta, \omega)\|_{B\left(X^{ \pm}(\nu(E) \omega), X^{ \pm}(\nu(E) \theta)\right)}=O\left(|E|^{-d+1}\right)
$$

as $E \rightarrow \pm \infty$.

$$
\lim _{E \rightarrow \pm \infty}\left\|\frac{F(E, \omega, \omega)}{|E|}-m^{ \pm}(\omega)\right\|_{B\left(X^{ \pm}(\nu(E) \omega), X^{ \pm}(\nu(E) \omega)\right)}=0 .
$$

Theorem 1.2. Suppose Assumption 1 and fix $\omega \in S^{2}$. Then

(1.8) $\lim _{E \rightarrow \pm \infty} \sup _{\substack{x \in(\mathcal{L}(E) \omega) \\|u|=1}}\left|\sigma(E, \omega, u)-2 \int_{\Pi_{\omega}}\left\{1-\cos \left(\frac{1}{c} \int_{-\infty}^{\infty} v_{ \pm \omega}(s \omega+\eta) d s\right)\right\} d \eta\right|=0$.

The latter theorem implies that the total scattering cross section has a limit independent of normalized initial internal states.

Finally, we show that the scalar potential $\phi$ and the magnetic field $\operatorname{rot} A$ can be reconstructed from the high energy behavior of the scattering amplitudes. To do so we fix an orthonormal basis $\left\{u_{j}^{ \pm}(p)\right\}_{{ }_{j}=1,2}$ of $X^{ \pm}(p)$ for each $p \in R^{3} \backslash\{0\}$ with 
the following properties throughout this paper:

Each $u_{j}^{ \pm}(p)$ is a $C^{4}$-valued continuous function of $p \in \boldsymbol{R}^{3} \backslash\{0\}$ and the limit

$$
u_{j}^{ \pm}(\infty, \omega):=\lim _{|E| \rightarrow \infty} u_{j}^{ \pm}(\nu(E) \omega)
$$

exist uniformly in $\omega \in S^{2}$.

It follows immediately that $u_{j}^{ \pm}(\infty, \omega)$ is continuous on $S^{2}$ and each $\left\{u_{j}^{ \pm}(\infty, \omega)\right\}_{j=1,2}$ forms an orthonormal basis of the space $X_{\infty}^{ \pm}(\omega):=\{u \in$ $\left.C^{4} \mid(\alpha \cdot \omega) u= \pm u\right\}$. If we define, for example, $\left\{\tilde{u}_{j}^{ \pm}(p)\right\}_{j=1,2}$ by

$$
\begin{aligned}
\left(\tilde{u}_{1}^{+}(p) \tilde{u}_{2}^{+}(p) \tilde{u}_{1}^{-}(p) \tilde{u}_{2}^{-}(p)\right) & =a_{+}(p) I_{4}-a_{-}(p) \beta \frac{\alpha \cdot p}{|p|}, \\
a_{ \pm}(p) & =\sqrt{\frac{1}{2} \pm \frac{m c^{2}}{2 \lambda(p)}},
\end{aligned}
$$

it is an orthonormal basis of $X^{ \pm}(p)$ and satisfies the above properties (see [Tha], p.9).

Let us define $J^{ \pm}(p): X^{ \pm}(p) \rightarrow C^{2}$ by

$$
J^{ \pm}(p)\left(\sum_{j=1}^{2} c_{j} u_{j}^{ \pm}(p)\right)=\left(\begin{array}{l}
c_{1} \\
c_{2}
\end{array}\right)
$$

Then the $2 \times 2$ matrix

$$
f(E, \theta, \omega)=J^{ \pm}(\nu(E) \theta) F(E, \theta, \omega) J^{ \pm}(\nu(E) \omega)^{-1}, \quad \pm E>m c^{2},
$$

is a representation of $F(E, \theta, \omega)$.

Let $\omega \in S^{2}$ and $\xi \in \Pi_{\omega}, \xi \neq 0$. Then we can take a family of $\theta(E) \in S^{2}$, $|E|>>1$ with $\lim _{|E| \rightarrow \infty} \nu(E)(\theta(E)-\omega)=\xi$.

Proposition 1.3. Suppose Assumption 1 with $\rho>3$. Let $\omega \in S^{2}$ and $\xi \in \Pi_{\omega}, \xi \neq 0$ and let $\{\theta(E)\}$ be as above. Then we have

$$
\lim _{E \rightarrow \pm \infty} \frac{f(E, \theta(E), \omega)}{|E|}=G_{ \pm \omega}(\xi)
$$

where

$$
G_{\omega}(\xi)=\frac{1}{2 \pi c i} \int_{\Pi_{\omega}} e^{-\imath \xi \cdot \eta}\left(e^{-(i / c) \int_{-\infty}^{\infty}(\phi(s \omega+\eta)-\omega \cdot c A(s \omega+\eta)) d s}-1\right) d \eta .
$$

The integral with respect to $\eta$ is absolutely convergent because of $\rho>3$.

Proposition 1.4. The scalar potential $\phi$ and the magnetic field $\operatorname{rot} A$ can be written in terms of the function $G_{\omega}(\xi)$ on $\left\{(\omega, \xi) \in S^{2} \times \boldsymbol{R}^{3} \mid \xi \in \Pi_{\omega}, \xi \neq 0\right\}$. 
Combining the above propositions, we immediately obtain the following result. The same result for Schrödinger operators with $A=0$ has been well-known since Faddeev $[\mathrm{F}]$.

Theorem 1.5. Suppose Assumption 1 with $\rho>3$. The scalar potential $\phi$ and the magnetic field rot $A$ can be determined by the high energy asymptotics of $f(E, \theta, \omega)$.

Remark. Here it should be noted that it is impossible to determine the magnetic potential $A$ itself because the scattering amplitude is invariant under the change $A \rightarrow A+\nabla \psi$ if $\phi$ is a rapidly decreasing smooth real-valued function.

In general, a Dirac operator $H$ has the form $H=H_{0}+V$, where $V$ is the multiplication operator by a $4 \times 4$ Hermitian matrix-valued function $V(x)$. Obviously, $V=V_{A \phi}$ in the electromagnetic case. In Sect. 2 the scattering amplitude and the total scattering cross section will be defined for a Dirac operator with a general potential $V(x)$, and a representation formula of the scattering amplitude will be given. In Sect. 3 it will be shown that Theorems 1.1, 1.2 and Proposition 1.3 follow immediately from more general results, i.e., Theorems $3.1,3.2$ and Proposition 3.3, respectively, and Proposition 1.4 is also proved. The limiting absorption principle, LAP, plays an important role in our proofs. Though the LAP for Dirac operators has been studied by several authors (see, e.g., [BMP], [PSU], $[\mathrm{V}],[\mathrm{Yam} 1]$ ), they discuss it in finite energy regions except for [PSU], and so it is needed to study the LAP for the high-energy range. In Sect. 4 we will give our result on the LAP for the high-energy range (Proposition 4.1). The proof can be carried out by combining the commutator method, due to Mourre, and a scaling argument in the same way as in the case of Schrödinger operators [Yaf2] Theorem 2. Thus, we give only a sketch of the proof in Sect.6. In Sect. 4 we will also show that the operator $e^{-i \nu(E) \omega \cdot x}(H-E-i 0)^{-1} e^{i \nu(E) \omega \cdot x}$ has a limit (in some sense) as $E \rightarrow \pm \infty$ and the limit is expressed by the resolvent of some first order differential operator (Corollary 4.4). Theorems 3.1, 3.2 and Proposition 3.3 will be proved in Sect. 5.

Recently, there are several works on scattering matrices for Dirac operators. See, for example, [BGU], [BGW], [BH] and the notes in [Tha].

\section{§ 2. Scattering Amplitude}

To define the scattering amplitude we need a spectral representation of $H_{0}$. Denote by $P^{ \pm}(p)$ the orthogonal projection from $C^{4}$ onto the subspace $X^{ \pm}(p)$ and write $\Sigma_{+}=\left(m c^{2},+\infty\right), \quad \Sigma_{-}=\left(-\infty,-m c^{2}\right), \Sigma=\Sigma_{-} \cup \Sigma_{+}$. For each $E \in \Sigma$ we define a positive constant $\mu(E)=c^{-3 / 2}\left(E^{2}\left(E^{2}-m^{2} c^{4}\right)\right)^{1 / 4}$ and à Hilbert space $\mathscr{H}(E)$ by a direct integral 


$$
\mathscr{H}(E)=\int_{S^{2}} \oplus X^{ \pm}(\nu(E) \omega) d \omega, \quad \pm E>m c^{2},
$$

(see $[\mathrm{BS}]$, ch.7 for the definition of the direct integrals). By definition, $\mathscr{H}(E)$, $\pm E>m c^{2}$, is a subspace of $L^{2}\left(S^{2} ; C^{4}\right)$ consisting of all elements $f$ satisfying $f(\omega) \in X^{ \pm}(\nu(E) \omega)$ for a.a. $\omega \in S^{2}$. We also define a unitary operator

$$
U_{H_{0}}: \mathscr{H} \rightarrow \mathscr{H}_{0}:=\int_{\Sigma} \oplus \mathscr{H}(E) d E
$$

by $\left(\left(U_{H_{0}} \psi\right)(E)\right)(\cdot)=\mu(E) P^{ \pm}(\nu(E) \cdot) \hat{\phi}(\nu(E) \cdot)$ for $\pm E>m c^{2}$, where $\hat{\phi}$ is the Fourier transform of $\phi$ :

$$
\hat{\phi}(p)=(2 \pi)^{-3 / 2} \int e^{-i x \cdot p} \phi(x) d x
$$

It is easy to see that $U_{H_{0}}$ gives a spectral representation of $H_{0}$ :

$$
U_{H_{0}} H_{0} U_{H_{0}}^{-1}=E,
$$

where the right-hand side is the multiplication operator by $E$.

Now let us consider a Dirac operator

$$
H=H_{0}+V
$$

where $V$ is the multiplication operator by an Hermitian matrix-valued function $V(x)=\left(V_{j k}(x)\right)_{1 \leq j, k \leq 4}$ on $\boldsymbol{R}^{3}$. We assume the following.

Assumption 2. There exist a real $\rho>2$ and an integer $d \geq 2$ such that each $V_{j k}, 1 \leq j, k \leq 4$, is in $C^{d}\left(\boldsymbol{R}^{3} ; \boldsymbol{C}\right)$ and obeys

$$
\left|\partial_{x}^{\gamma} V_{j k}(x)\right| \leq C(1+|x|)^{-\rho}, \quad|\gamma| \leq d
$$

for some $C>0$.

Obviously the potential $V=V_{A \phi}$, defined in the previous section, satisfies Assumption 2 if $A$ and $\phi$ satisfy Assumption 1. Under Assumption 2, $H$ is a self-adjoint operator with domain $D(H)=D\left(H_{0}\right)$, and moreover the wave operators

$$
W_{ \pm}=s-\lim _{t \rightarrow \pm \infty} \exp (i t H) \exp \left(-i t H_{0}\right) \quad \in \boldsymbol{B}(\mathscr{H})
$$

exist and are asymptotically complete: 


$$
\text { Range } W_{ \pm}=\mathscr{H}_{a c}(H)
$$

where $\mathscr{H}_{a c}(H)$ denotes the absolutely continuous subspace of $\mathscr{H}$ (see, e.g., [Tha]). It is also known that there is no eigenvalue in $\Sigma$ under Assumption 2 ([Yam 1], Proposition 2.5). It follows from (2.2) and (2.3) that the scattering operator $S=W_{+}^{*} W_{-}$is a unitary operator on $\mathscr{H}$. On the other hand, it follows from the definition of the wave operators that $\exp (i t H) W_{ \pm}=W_{ \pm} \exp \left(i t H_{0}\right)$ for all $t \in \boldsymbol{R}$, and so, $S$ commutes with $H_{0}$ :

$$
\exp \left(i t H_{0}\right) S=S \exp \left(i t H_{0}\right), \quad t \in \boldsymbol{R} .
$$

This fact guarantees that $U_{H_{0}} S U_{H_{0}}^{-1}$ is a decomposable operator on $\mathscr{H}_{0}$ :

$$
U_{H_{0}} S U_{H_{0}}^{-1}=\int_{\Sigma} \oplus S(E) d E
$$

where $S(E)$, called the scattering matrix at energy $E$, is a unitary operator on $\mathscr{H}(E)$. Under Assumption 2, $S(E)-I$ is of Hilbert-Schmidt class for each $E \in \Sigma$ and its integral kernel is denoted by $T(E, \theta, \omega) \in \boldsymbol{B}\left(X^{ \pm}(\nu(E) \omega), X^{ \pm}(\nu(E) \theta)\right)$, where $(E, \theta, \omega) \in \Sigma_{ \pm} \times S^{2} \times S^{2}$. The scattering amplitude with energy $E$, incident direction $\omega$ and scattering direction $\theta$ is defined by

$$
F(E, \theta, \omega)=-2 \pi i \nu(E)^{-1} T(E, \theta, \omega),
$$

which is well-defined for each $(E, \theta, \omega)$ if $\rho>3$. For $u \in X^{ \pm}(\nu(E) \omega)$ with $|u|=1$, the differential cross section is defined by $|F(E, \theta, \omega) u|^{2}$ and the total scattering cross section is defined by (1.4), which is well-defined for each $(E, \omega)$ and $u$ for $\rho>2$ (see Proposition 2.1 below).

We give a representation formula of $f(E, \theta, \omega)$ defined by (1.9). For $s \in \boldsymbol{R}$ we define

$$
L_{s}^{2}\left(\boldsymbol{R}^{3} ; \boldsymbol{C}^{4}\right)=\left\{f \in L_{l o c}^{2}\left(\boldsymbol{R}^{3} ; \boldsymbol{C}^{4}\right): \begin{array}{l}
\mid \\
\mid
\end{array}\left\|_{L_{s}^{2}}=\right\|\left(1+|x|^{2}\right)^{s / 2} f \|_{L_{2}\left(R^{3} ; C^{4}\right)}<\infty\right\}
$$

and $\boldsymbol{B}_{s}=\boldsymbol{B}\left(L_{s}^{2}\left(\boldsymbol{R}^{3} ; \boldsymbol{C}^{4}\right), L_{-s}^{2}\left(\boldsymbol{R}^{3} ; \boldsymbol{C}^{4}\right)\right)$. Let $R(z):=(H-\boldsymbol{z})^{-1}$ for $\operatorname{Im} \boldsymbol{z} \neq 0$. Then, it is known that the following norm limits exist in $\boldsymbol{B}_{s}, s>1 / 2$, uniformly for $E$ in any compact set of $\Sigma$ :

$$
R(E \pm i 0)=\lim _{\varepsilon \vee 0} R(E \pm i \varepsilon)
$$

and that $R(E \pm i 0)$ is a $\boldsymbol{B}_{s}$-valued continuous function of $E$. This fact is called the limiting absorption principle (see, e.g., [BMP], [V], [Yam1]). We define an operator $Q(E):=-V+V R(E+i 0) V$ for each $E \in \Sigma$ and a $4 \times 4$ matrix $\hat{Q}(E, \theta, \omega)=\left(\hat{Q}_{j k}(E, \theta, \omega)\right)$ by 


$$
\hat{Q}_{j k}(E, \theta, \omega):=\left(Q(E) e_{0}(\nu(E) \omega, \cdot) \chi_{k}, e_{0}(\nu(E) \theta, \cdot) \chi_{j}\right)_{L^{2}\left(R^{3} ; C^{4}\right)}
$$

where $e_{0}(p, x)=e^{i p \cdot x}$ and $\left\{\chi_{j}\right\}_{1 \leq j \leq 4}$ is the canonical basis of $C^{4}: \chi_{1}={ }^{t}(1,0,0,0)$, $\cdots, \chi_{4}={ }^{t}(0,0,0,1)$. The following result is obtained by a usual way in scattering theory, and we give only a sketch of the proof.

Proposition 2.1. Suppose Assumption 2. Then $S(E)-I$ is of Hilbert-Schmidt class for each $E \in \Sigma$, and $f(E, \theta, \omega)$ defined in (1.9) has the following representation.

(2.6) $f(E, \theta, \omega)$

$$
=\left(2 \pi c^{2}\right)^{-1}|E|\left(u_{1}^{ \pm}(\nu(E) \theta) u_{2}^{ \pm}(\nu(E) \theta)\right)^{*} \hat{Q}(E, \theta, \omega)\left(u_{1}^{ \pm}(\nu(E) \omega) u_{2}^{ \pm}(\nu(E) \omega)\right)
$$

for $E \in \Sigma_{ \pm}$, where $\left(u_{1}^{ \pm}(\nu(E) \theta) u_{2}^{ \pm}(\nu(E) \theta)\right)$ is the $4 \times 2$ matrix with the $j$-th column vector $u_{j}^{ \pm}(\nu(E) \theta)$. In particular, $f(E, \theta, \omega)$ has the following properties:

(i) Each component of $f(E, \cdot, \omega)$ is an $L^{2}\left(S^{2}\right)$-valued continuous function of $(E, \omega) \in \Sigma \times S^{2}$.

(ii) $f(E, \theta, \omega)-f(E, \omega, \theta)^{*}$ is continuous in $(E, \theta, \omega) \in \Sigma \times S^{2} \times S^{2}$.

Moreover, if $\rho>3$, then $S(E)-I$ is of trace class for each $E \in \Sigma$, and $f(E, \theta, \omega)$ is continuous in $(E, \theta, \omega) \in \Sigma \times S^{2} \times S^{2}$.

Sketch of the proof. We define $U_{H_{0}}(E): L_{s}^{2}\left(\boldsymbol{R}^{3}, C^{4}\right) \rightarrow \mathscr{H}(E), s>1 / 2$, by $\left(U_{H_{0}}(E) \psi\right)(\cdot)=\left(\left(U_{H_{0}} \psi\right)(E)\right)(\cdot)$. Then the following relation is verified

$$
U_{H_{0}}(E)^{*} U_{H_{0}}(E)=\frac{1}{2 \pi i}\left(\left(H_{0}-E-i 0\right)^{-1}-\left(H_{0}-E+i 0\right)^{-1}\right) .
$$

Taking account of this relation, we can derive

$$
S(E)-I=2 \pi i U_{H_{0}}(E) Q(E) U_{H_{0}}(E) * \text { for a.a. } E
$$

in a usual way (see, for example, [Yaf1], p.94) and verify that the right-hand side is of Hilbert-Schmidt class if $\rho>2$, and of trace class if $\rho>3$. Therefore, from the definition of $U_{H_{0}}(E)$ it follows that

$$
F(E, \theta, \omega)=\left.\left(2 \pi c^{2}\right)^{-1}|E| P^{ \pm}(\nu(E) \theta) \hat{Q}(E, \theta, \omega)\right|_{X^{ \pm}(\nu(E) \omega)}
$$

where $\left.\right|_{X^{ \pm}(\nu(E) \omega)}$ denotes the restriction to $X^{ \pm}(\nu(E) \omega)$. Hence, we obtain (2.6) by (1.9). The properties of $f(E, \theta, \omega)$ follow from those of $\hat{Q}(E, \theta, \omega)$ discussed below.

We see that $V \in \boldsymbol{B}\left(L_{-s}^{2}, L_{t}^{2}\right)$ for $s+t \leq \rho$ and $V R(R \pm i 0) V \in \boldsymbol{B}_{-s}$ for $s<$ $\rho-(1 / 2)$ by Assumption 2 and the limiting absorption principle. Since $\chi_{k} \in L_{s}^{2}$ for any $s<-3 / 2$, the $j$-component of $Q(E) e_{0}(\nu(E) \omega, \cdot) \chi_{k}$ is an $L_{s}\left(\boldsymbol{R}^{3}\right)$-valued 
continuous function of $(E, \omega)$ for some $s>1 \%$. Thus, noting that $\hat{Q}_{j k}(E, \cdot, \omega)$ is the restriction of the Fourier transform of the function to the sphere $\left\{\xi \in \boldsymbol{R}^{3}|| \xi \mid=\nu(E)\right\}$, we see that $\hat{Q}_{j k}(E, \cdot, \omega)$ is an $L^{2}\left(S^{2}\right)$-valued continuous function of $(E, \omega) \in \Sigma \times S^{2}$. On the other hand, by the equality

$$
\begin{aligned}
& \hat{Q}(E, \theta, \omega)-\hat{Q}(E, \omega, \theta)^{*} \\
& \quad=\left(\left(V(R(E+i 0)-R(E-i 0)) V e_{0}(\nu(E) \omega, \cdot) \chi_{k}, e_{0}(\nu(E) \theta, \cdot) \chi_{j}\right)_{L^{2}\left(R^{3} ; C^{4}\right)}\right),
\end{aligned}
$$

we can verify that it is continuous in $(E, \theta, \omega) \in \Sigma \times S^{2} \times S^{2}$. Moreover, it is easily seen that $\hat{Q}(E, \theta, \omega)$ is also continuous $(E, \theta, \omega) \in \Sigma \times S^{2} \times S^{2}$ if $\rho>3$.

\section{$\S 3$. General Potentials}

Theorems 1.1, 1.2 and Proposition 1.3 follow from more general results below (Theorems 3.1, 3.2, Proposition 3.3). To state these results we prepare some notations. For each $\omega \in S^{2}$, define the $2 \times 2$ matrix

$$
V_{\omega}^{ \pm}(x)=K^{ \pm}(\omega)^{*} V(x) K^{ \pm}(\omega)
$$

where $K^{ \pm}(\omega)=\left(u_{1}^{ \pm}(\infty, \omega) u_{2}^{ \pm}(\infty, \omega)\right)$ is the $4 \times 2$ matrix with the $j$-th column vector $u_{j}^{ \pm}(\infty, \omega)$. For each $\eta \in \Pi_{\omega}$ let $U_{\omega}^{ \pm}(t, \eta)$ be a $2 \times 2$ matrix-valued function on $\boldsymbol{R}$ satisfying the following equation:

$$
\begin{aligned}
& c i \frac{d}{d t} U_{\omega}^{ \pm}(t, \eta)=V_{\omega}^{ \pm}(t \omega+\eta) U_{\omega}^{ \pm}(t, \eta), \\
& \lim _{t \rightarrow-\infty} U_{\omega}^{ \pm}(t, \eta)=I_{2} .
\end{aligned}
$$

It is easily verified that under Assumption 2 this equation has a unique solution and that the solution is a unitary matrix for each $t$ and has a limit as $t \rightarrow+\infty$ :

$$
U_{\omega}^{ \pm}(+\infty, \eta):=\lim _{t \rightarrow+\infty} U_{\omega}^{ \pm}(t, \eta)
$$

Moreover, we have the following estimate easily:

$$
\text { li } U_{\omega}^{ \pm}(+\infty, \eta)-I_{2} \| \leq C(1+|\eta|)^{-\rho+1} \text {. }
$$

Let $\operatorname{Re} U=\left(U+U^{*}\right) / 2$. Then $\left(U-I_{2}\right)\left(U^{*}-I_{2}\right)=2-2 \operatorname{Re} U$, and hence

$$
\left\|\operatorname{Re} U_{\omega}^{ \pm}(+\infty, \eta)-I_{2}\right\| \leq C(1+|\eta|)^{-2 \rho+2} .
$$


Theorem 3.1. Suppose Assumption 2 with $\rho>3$. Let $\omega \in S^{2}$.

(i) Let $K$ be an arbitrary compact set in $S^{2} \backslash\{\omega\}$. Then

$$
\sup _{\theta \in K}\|F(E, \theta, \omega)\|_{B\left(X^{ \pm}(\nu(E) \omega), X^{ \pm}(\nu(E) \theta)\right)}=O\left(|E|^{-d+1}\right)
$$

as $E \rightarrow \pm \infty$.

(ii) Let

$$
M^{ \pm}(\omega):=\frac{1}{2 \pi c i} \int_{\Pi_{\omega}}\left(U_{\omega}^{ \pm}(+\infty, \eta)-I_{2}\right) d \eta
$$

Then

$$
\begin{aligned}
& \lim _{E \rightarrow \pm \infty}\left\|\frac{F(E, \omega, \omega)}{|E|}-J^{ \pm}(\nu(E) \omega)^{-1} M^{ \pm}(\omega) J^{ \pm}(\nu(E) \omega)\right\|_{B\left(X^{ \pm}(\nu(E) \omega), X^{ \pm}(\nu(E) \omega)\right)} \\
= & \lim _{E \rightarrow \pm \infty}\left\|\frac{f(E, \omega, \omega)}{|E|}-M^{ \pm}(\omega)\right\|_{B\left(C^{2}\right)}=0 .
\end{aligned}
$$

Next we consider the total scattering cross section. A normalized internal state $u \in X^{ \pm}(\nu(E) \omega)$ can be written as $u=\sum_{j=1}^{2} c_{k} u_{k}^{ \pm}(\nu(E) \omega), \pm E>m c^{2}$ with $c_{1}, c_{2} \in C,\left|c_{1}\right|^{2}+\left|c_{2}\right|^{2}=1$. Let $f_{j k}(E, \theta, \omega)$ denote the $(j, k)$-component of $f(E, \theta, \omega)$. Then

$$
\begin{aligned}
\sigma(E, \omega, u) & =\sum_{j=1}^{2} \int_{S^{2}}\left|\sum_{k=1}^{2} c_{k} f_{j k}(E, \theta, \omega)\right|^{2} d \theta \\
& =\sum_{k=1}^{2}\left|c_{k}\right|^{2} \sigma_{k}(E, \omega)+2 \operatorname{Re}\left(c_{1} \bar{c}_{2} \sigma_{21}(E, \omega)\right),
\end{aligned}
$$

where

$$
\begin{aligned}
& \sigma_{k}(E, \omega)=\sum_{j=1}^{2} \int_{S^{2}}\left|f_{j k}(E, \theta, \omega)\right|^{2} d \theta, \quad k=1,2, \\
& \sigma_{21}(E, \omega)=\sum_{j=1}^{2} \int_{S^{2}} f_{j 1}(E, \theta, \omega) \overline{f_{j 2}(E, \theta, \omega)} d \theta .
\end{aligned}
$$

Theorem 3.2. Suppose Assumption 2. Let $\omega \in S^{2}$. Then

$$
\begin{aligned}
& \lim _{E \rightarrow \pm \infty} \sigma_{k}(E, \omega)=2 \int_{\Pi_{\omega}}\left\{1-\operatorname{Re} U_{\omega}^{ \pm}(+\infty, \eta)_{k k}\right\} d \eta, \quad k=1,2, \\
& \lim _{E \rightarrow \pm \infty} \sigma_{21}(E, \omega)=-\int_{\Pi_{\omega}}\left\{U_{\omega}^{ \pm}(+\infty, \eta)_{21}+\overline{U_{\omega}^{ \pm}(+\infty, \eta)_{12}}\right\} d \eta,
\end{aligned}
$$

where $U_{\omega}^{ \pm}(+\infty, \eta)_{j k}$ is the $(j, k)$-component of $U_{\omega}^{ \pm}(+\infty, \eta)$.

Proposition 3.3. Suppose Assumption 2 with $\rho>3$. Let $\omega \in S^{2}$ and $\xi \in \Pi_{\omega}$, 
$\xi \neq 0$, and let $\{\theta(E)\}$ be a family of unit vectors in $\boldsymbol{R}^{3}$ such that $\nu(E)(\theta(E)-\omega) \rightarrow \xi$ as $|E| \rightarrow \infty$. Then

$$
\lim _{E \rightarrow \pm \infty} \frac{f(E, \theta(E), \omega)}{E}=\frac{1}{2 \pi c i} \int_{\Pi_{\omega}} e^{-i \xi \cdot \eta}\left(U_{\omega}^{ \pm}(+\infty, \eta)-I_{2}\right) d \eta
$$

Accepting Theorems 3.1, 3.2 and Proposition 3.3, we prove Theorems 1.1, 1.2 and Proposition 1.3.

Proofs of Theorems 1.1, 1.2 and Proposition 1.3. We first prove that

$$
K^{ \pm}(\omega)^{*}(\alpha \cdot A) K^{ \pm}(\omega)= \pm(\omega \cdot A) I_{2}
$$

Indeed, the $(j, k)$-component of the matrix $K^{ \pm}(\omega) *(\alpha \cdot A) K^{ \pm}(\omega)$ can be written as follows:

$$
\begin{aligned}
\left((\alpha \cdot A) u_{k}^{ \pm}, u_{\jmath}^{ \pm}\right)_{c^{4}} & =\left((\alpha \cdot A)( \pm \alpha \cdot \omega) u_{k}^{ \pm}, u_{\jmath}^{ \pm}\right)_{c^{4}} \\
& =\left((\alpha \cdot A) u_{k}^{ \pm},( \pm \alpha \cdot \omega) u_{\jmath}^{ \pm}\right)_{c^{4}}
\end{aligned}
$$

because each $u_{j}^{ \pm}=u_{j}^{ \pm}(\infty, \omega)$ belongs to $X_{\infty}^{ \pm}(\omega)$. On the other hand, taking account of (1.1), we get the well-known formula

$$
(\alpha \cdot A)(\alpha \cdot \omega)+(\alpha \cdot \omega)(\alpha \cdot A)=2 \omega \cdot A .
$$

Thus, (3.12) follows immediately. Substituting $V=V_{A \phi}$ in (3.1) and using (3.12), we see that $V_{\omega}^{ \pm}(x)$ is a scalar function:

$$
V_{\omega}^{ \pm}(x)=v_{ \pm \omega}(x) I_{2}, \quad v_{ \pm \omega}(x):=\phi(x) \mp \omega \cdot c A(x),
$$

and

$$
U_{\omega}^{ \pm}(t, \eta)=\exp \left(-\frac{i}{c} \int_{-\infty}^{t} v_{ \pm \omega}(s \omega+\eta) d s\right) I_{2}
$$

Therefore,

$$
\begin{gathered}
U_{\omega}^{ \pm}(+\infty, \eta)_{12}=U_{\omega}^{ \pm}(+\infty, \eta)_{21}=0, \\
\operatorname{Re} U_{\omega}^{ \pm}(+\infty, \eta)_{k k}=\cos \left(\frac{1}{c} \int_{-\infty}^{\infty} v_{ \pm \omega}(s \omega+\eta) d s\right)
\end{gathered}
$$

and $M^{ \pm}(\omega)=m^{ \pm}(\omega) I_{2}$ (see (1.5)). Hence, Theorems 1.1, 1.2 and Proposition 1.3 immediately follow from Theorems 3.1, 3.2 and Proposition 3.3, respectively.

Next we give the proof of Proposition 1.4. 
Proof of Proposition 1.4. Since $G_{\omega}(\xi)$ is the Fourier transform of a function const. $\left(e^{-(i / c) \int_{-\infty}^{\infty}(\phi(s \omega+\eta)-\omega \cdot c A(s \omega+\eta)) d s}-1\right)$ of $\eta \in \Pi_{\omega}$, and since $\int_{-\infty}^{\infty}(\phi(s \omega+\eta)-$ $\omega \cdot c A(s \omega+\eta)) d s$ is continuous in $\eta \in \Pi_{\omega}$ and goes to zero as $|\eta| \rightarrow \infty$, the function $\int_{-\infty}^{\infty}(\phi(s \omega+\eta)-\omega \cdot c A(s \omega+\eta)) d s$ can be determined by the function $G_{\omega}(\xi), \xi \in$ $\Pi_{\omega}$. Replacing $\omega$ by $-\omega$, we also obtain $\int_{-\infty}^{\infty}(\phi(s \omega+\eta)+\omega \cdot c A(s \omega+\eta)) d s$ from $G_{-\omega}(\xi)$. Thus, both $\int_{-\infty}^{\infty} \phi(s \omega+\eta) d s$ and $\int_{-\infty}^{\infty} \omega \cdot A(s \omega+\eta) d s$ are determined by $G_{ \pm \omega}(\xi), \xi \in \Pi_{\omega}$. Taking account of the Fourier transform on $\boldsymbol{R}^{3}$

$$
\hat{\phi}(\xi)=(2 \pi)^{-3 / 2} \int_{\Pi_{\omega}} e^{-\imath \xi \cdot \eta}\left(\int_{-\infty}^{\infty} \phi(s \omega+\eta) d s\right) d \eta, \quad \xi \in \Pi_{\omega},
$$

we see that $\phi$ is determined by the function $G_{\omega}(\xi)$ of $(\omega, \xi)$ with $\omega \in S^{2}, \xi \in \Pi_{\omega}$, because $\omega$ can move on the whole sphere. To represent the magnetic field $\operatorname{rot} A$ in terms of $\int_{-\infty}^{\infty} \omega \cdot A(t \omega+\eta) d t, \omega \in S^{2}, \eta \in \Pi_{\omega}$, we prepare the following.

Lemma 3.4. Let $\left\{\theta, \omega, \omega^{\prime}\right\}$ be an orthonormal basis of $\boldsymbol{R}^{3}$ with $\omega^{\prime}=\theta \times \omega$, and let

$$
a_{\omega}(\eta):=\int_{-\infty}^{\infty} \omega \cdot A(t \omega+\eta) d t, \quad \eta \in \Pi_{\omega} .
$$

Then

$$
\left(\theta \cdot \operatorname{rot} A \hat{)}(\xi)=(2 \pi)^{-3 / 2} i\left(\xi \cdot \omega^{\prime}\right) \int_{\Pi_{\omega}} e^{-i \xi \cdot \eta} a_{\omega}(\eta) d \eta\right.
$$

for $\xi \in \Pi_{\omega}$, where the left-hand side is the Fourier transform of the function $\theta \cdot \operatorname{rot} A$ on $R^{3}$.

Proof of Lemma 3.4. Taking account of $\rho>3$, we have by Stokes' theorem

$$
a_{\omega}\left(\lambda \omega^{\prime}+\mu \theta\right)-a_{\omega}\left(\lambda^{\prime} \omega^{\prime}+\mu \theta\right)=\int_{\lambda}^{\lambda^{\prime}} d s \int_{-\infty}^{\infty}(\theta \cdot \operatorname{rot} A)\left(t \omega+s \omega^{\prime}+\mu \theta\right) d t
$$

for $\lambda, \lambda^{\prime}, \mu \in \boldsymbol{R}$. Thus,

$$
\frac{\partial}{\partial \lambda} a_{\omega}\left(\lambda \omega^{\prime}+\mu \theta\right)=-\int_{-\infty}^{\infty}(\theta \cdot \operatorname{rot} A)\left(t \omega+\lambda \omega^{\prime}+\mu \theta\right) d t
$$

and so,

$$
\begin{aligned}
-(2 \pi)^{3 / 2}\left(\theta \cdot \operatorname{rot} A \hat{)}\left(\xi_{1} \omega^{\prime}+\xi_{2} \theta\right)\right. & =\iint e^{-i \xi_{1} \lambda-i \xi_{2} \mu} \frac{\partial}{\partial \lambda} a_{\omega}\left(\lambda \omega^{\prime}+\mu \theta\right) d \lambda d \mu \\
& =-i \xi_{1} \iint e^{-i \xi_{1} \lambda-i \xi_{2} \mu} a_{\omega}\left(\lambda \omega^{\prime}+\mu \theta\right) d \lambda d \mu,
\end{aligned}
$$


where we have used integration by parts at the second step. We have thus proved the lemma.

Since, for fixed $\theta \in S^{2}$, the union of $\left\{\Pi_{\omega} \mid \omega \in S^{2} \cap \Pi_{\theta}\right\}$ coincides with the whole space $R^{3}$, the function $\theta \cdot \operatorname{rot} A(x)$ is determined by $\left\{a_{\omega}(\eta) \mid \omega \in S^{2}, \eta \in \Pi_{\omega}\right\}$ by virtue of the above lemma. Consequently, $\operatorname{rot} A(x)$ is determined by this set for $\theta$ is arbitrary, and Proposition 1.4 has been proved.

\section{§ 4. High Energy Behavior of The Resolvent}

The following assumption on potentials is sufficient for the limiting absorption principle at the high-energy.

Assumption 3. $V(x)$ is an Hermitian matrix-valued $C^{2}$ function on $\boldsymbol{R}^{3}$ such that all $\left|(x \cdot \nabla)^{\ell} V_{j k}(x)\right|$ are bounded on $R^{3}$ for $\ell=0,1,2$.

Proposition 4.1. There exists $E_{0}>0$ such that for any $s$ with $1 / 2<s \leq 1$ the following estimates are valid:

$$
\begin{gathered}
\sup _{\substack{0<\varepsilon<1 \\
|E| \geq E_{0}}}\|R(E \pm i \varepsilon)\|_{B_{s}}<+\infty, \\
\sup _{|E| \geq E_{0}}\left\|R(E \pm i \varepsilon)-R\left(E \pm i \varepsilon^{\prime}\right)\right\|_{B_{s}} \leq C_{s}\left|\varepsilon-\varepsilon^{\prime}\right|^{\alpha}, \quad 0<\varepsilon, \varepsilon^{\prime}<1
\end{gathered}
$$

for some $C_{s}>0$, where $\alpha=(s-(1 / 2)) /(s+(1 / 2))$. In particular, the norm limits $R(E \pm i 0): \lim _{\varepsilon \downarrow 0} R(E \pm i \varepsilon)$ exist in $\boldsymbol{B}_{s}$ uniformly in $|E| \geq E_{0}$.

Remark 1. Let $W(x)=\left(W_{\jmath k}(x)\right)$ be a $4 \times 4$ Hermitian matrix-valued $C^{1}$ function such that $(1+|x|)^{\rho}\left|W_{j k}(x)\right|$ and $\left|\nabla W_{j k}(x)\right|$ are bounded for some $\rho>1$ and let $V(x)=\kappa W(x), \kappa \in R$, then the same results have been obtained by Pladdy, Saitō and Umeda [PSU] for small $\kappa$ 's.

Remark 2. Though the resolvent estimate for any compact energy range in $\Sigma$ has been already proved by many authors (see, e.g., [BMP], [V], [Yam1]), the estimate for the high energy range $(|E|>>1)$ is, so far as the author knows, proved in only [PSU] above. On the other hand, in the case of Schrödinger operators, Yafaev [Yaf2] has obtained high-energy resolvent estimates by combining the commutator method, due to Mourre ( $[\mathrm{CFKS}],[\mathrm{M}]$ ), and a scaling argument (see also $[\mathrm{J}]$ ). His argument is applicable to our case, and we can prove Proposition 4.1 in the same way as [Yaf2]. So, we will give only a sketch of the proof in Sect.6. 
Next we study the asymptotic behavior of $R(E+i 0)$ as $E \rightarrow \pm \infty$. For proving Propositions 4.2, 4.3 below, the following assumption on the Hermitian matrixvalued function $V(x)$ is sufficient:

Assumption 4. Each $V_{j k}, 1 \leq j, k \leq 4$, is in $C^{2}\left(\boldsymbol{R}^{3} ; \boldsymbol{C}\right)$ and

$$
\sup _{x \in R^{3}}\left|\partial_{x}^{\gamma} V_{j k}(x)\right| \leq C, \quad|\gamma| \leq 2
$$

for some $C>0$.

Define an operator $L(\omega), \omega \in S^{2}$, in $L^{2}\left(\boldsymbol{R}^{3} ; \boldsymbol{C}^{2}\right)$ by

$$
L(\omega)=c \omega \cdot D+K_{0}(\omega)^{*} V K_{0}(\omega), \quad K_{0}(\omega)=\frac{1}{\sqrt{2}}\left(\begin{array}{c}
I_{2} \\
\sigma \cdot \omega
\end{array}\right) .
$$

It is easily seen that the operator $K_{0}( \pm \omega)$ can be regarded as a unitary operator from $L^{2}\left(\boldsymbol{R}^{3} ; \boldsymbol{C}^{2}\right)$ onto $L^{2}\left(\boldsymbol{R}^{3} ; X_{\infty}^{ \pm}(\omega)\right)$ as well as from $\boldsymbol{C}^{2}$ onto $X_{\infty}^{ \pm}(\omega)$. The operator $L(\omega)$ is a self-adjoint operator with domain

$$
D(L(\omega))=\left\{f \in L^{2}\left(\boldsymbol{R}^{3} ; \boldsymbol{C}^{2}\right) \mid \omega \cdot D f \in L^{2}\left(\boldsymbol{R}^{3} ; \boldsymbol{C}^{2}\right)\right\}
$$

Proposition 4.2. Suppose Assumption 4. Let $\varepsilon>0, \omega \in S^{2}$ and $f \in L^{2}\left(\boldsymbol{R}^{3} ; \boldsymbol{C}^{4}\right)$. Then one has

$$
s-\lim _{E \rightarrow \pm \infty} e^{-\imath \nu(E) \omega \cdot x} R(E+i \varepsilon) e^{i \nu(E) \omega \cdot x} f=K_{0}( \pm \omega)(L( \pm \omega)-i \varepsilon)^{-1} K_{0}( \pm \omega)^{*} f
$$

in $L^{2}\left(\boldsymbol{R}^{3} ; \boldsymbol{C}^{4}\right)$, where $K_{0}( \pm \omega)^{*}$ is the adjoint matrix of $K_{0}( \pm \omega)$.

The proof is given at the end of this section.

The limiting absorption principle for $L(\omega)$ also holds:

Proposition 4.3. Suppose Assumption 4. Let $\omega \in S^{2}$ and $s>1 / 2$. Then

$$
\sup _{0<\varepsilon<1}\left\|(L(\omega)-i \varepsilon)^{-1}\right\|_{B_{s}^{\prime}}<+\infty,
$$

and the norm limit $(L(\omega)-i 0)^{-1}:=\lim _{\varepsilon \downarrow 0}(L(\omega)-i \varepsilon)^{-1}$ exists in $\boldsymbol{B}_{s}^{\prime}$, where $\boldsymbol{B}_{s}^{\prime}:=\boldsymbol{B}\left(L_{s}^{2}\left(\boldsymbol{R}^{3} ; \boldsymbol{C}^{2}\right), L_{-s}^{2}\left(\boldsymbol{R}^{3} ; \boldsymbol{C}^{2}\right)\right)$.

Proof. We use the commutator method by Mourre (see, [M], [PSS], [CFKS]). Let $A$ be the multiplication operator by $\omega \cdot x$. Since $i[L(\omega), A]=c$ on the space of rapidly decreasing functions, we can adopt $A$ as a conjugate operator for $L(\omega)$ to obtain the desired results. The detail is ommited. 
Combining Propositions 4.1, 4.2 and 4.3, we obtain the following result immediately.

Corollary 4.4. Suppose Assumptions 3 and 4. Let $s>1 / 2$ and $f \in L_{s}^{2}\left(\boldsymbol{R}^{3} ; \boldsymbol{C}^{4}\right)$. Then one has

$$
s-\lim _{E \rightarrow \pm \infty} e^{-\imath \nu(E) \omega \cdot x} R(E+i 0) e^{\imath \nu(E) \omega \cdot x} f=K_{0}( \pm \omega)(L( \pm \omega)-i 0)^{-1} K_{0}( \pm \omega)^{*} f
$$

in $L_{-s}^{2}\left(\boldsymbol{R}^{3} ; \boldsymbol{C}^{4}\right)$.

Remark 1. Note that the corollary implies

$$
\lim _{|E| \rightarrow \pm \infty} \inf \|R(E \pm i 0)\|_{B_{s}} \neq 0
$$

for any $s>1 / 2$. In the case of the free Dirac operator, $V=0$, this has been already pointed out by Yamada [Yam2] (see also [Yam3]). On the other hand, in the case of Schrödinger operators, the following estimate is well-known for any $s>1 / 2$ :

$$
\left\|(-\Delta+v-E \pm i 0)^{-1}\right\|_{B\left(L_{s}^{2}, L_{-s}^{2}\right)}=O\left(E^{-1 / 2}\right), \quad E \rightarrow+\infty,
$$

for a large class of potentials $v$, and this estimate (4.4) plays an important role when one reconstructs $v$ from the high energy behavior of scattering amplitudes (see, e.g., $[\mathrm{F}],[\mathrm{N}],[\mathrm{S} 1],[\mathrm{S} 2]$ ). Since a similar estimate such as (4.4) dose not hold in the case of Dirac operators, we need a more detailed information of the high energy behavior of the resolvent such as Corollary 4.4 to consider similar reconstruction problems.

Remark 2. Denote by $P_{\infty}^{ \pm}(\omega)$ the orthogonal projection from $C^{4}$ onto $X_{\infty}^{ \pm}(\omega)$ and by $E_{\infty}^{ \pm}(\omega)$ the embedding from $X_{\infty}^{ \pm}(\omega)$ into $C^{4}$. We can regard them as the orthogonal projection from $L^{2}\left(\boldsymbol{R}^{3} ; \boldsymbol{C}^{4}\right)$ onto $L^{2}\left(\boldsymbol{R}^{3} ; X_{\infty}^{ \pm}(\omega)\right)$ and the embedding from $L^{2}\left(\boldsymbol{R}^{3} ; X_{\infty}^{ \pm}(\omega)\right)$ into $L^{2}\left(\boldsymbol{R}^{3} ; \boldsymbol{C}^{4}\right)$, respectively. Define an operator $L^{ \pm}(\omega)$ in $L^{2}\left(\boldsymbol{R}^{3} ; X_{\infty}^{ \pm}(\omega)\right)$ by

$$
L^{ \pm}(\omega)=c \omega \cdot D+P_{\infty}^{ \pm}(\omega) V(x) E_{\infty}^{ \pm}(\omega)
$$

which is the restriction of the operator $c \omega \cdot D+V(x)$ in $L^{2}\left(\boldsymbol{R}^{3} ; C^{4}\right)$ to $L^{2}\left(\boldsymbol{R}^{3} ; X_{\infty}^{ \pm}(\omega)\right)$. If we identify $C^{2}$ with $X_{\infty}^{ \pm}(\omega)$ by $K_{0}( \pm \omega)$, then $L( \pm \omega)$ is identified with $L^{ \pm}(\omega)$. Moreover, we can easily see that

$$
K_{0}( \pm \omega)(L( \pm \omega)-i 0)^{-1} K_{0}( \pm \omega)^{*}=E_{\infty}^{ \pm}(\omega)\left(L^{ \pm}(\omega)-i 0\right)^{-1} P_{\infty}^{ \pm}(\omega)
$$

Since $\left\{u_{j}^{ \pm}(\infty, \omega)\right\}_{j=1,2}$ is an orthonormal basis of $X_{\infty}^{ \pm}(\omega)$, we also have 
(4.7) $K_{0}( \pm \omega)(L( \pm \omega)-i 0)^{-1} K_{0}( \pm \omega)^{*}=K^{ \pm}(\omega)\left(c \omega \cdot D+V_{\omega}^{ \pm}-i 0\right)^{-1} K^{ \pm}(\omega)^{*}$.

Proof of Proposition 4.2. In this proof we fix $\omega \in S^{2}$ and $\varepsilon>0$, and write $\nu=\nu(E)$ (see (1.3)) and $\|\cdot\|=\|\cdot\|_{B\left(L^{2}\left(R^{3} ; C^{2}\right)\right)}$ for simplicity. First note that

$$
e^{-i \nu \omega \cdot x}(H-E-i \varepsilon) e^{i \nu \omega \cdot x}=\left(\begin{array}{ll}
K & L \\
L^{*} & M
\end{array}\right)
$$

where

$$
\begin{aligned}
& K=m c^{2}+U_{11}-E-i \varepsilon, \quad M=-m c^{2}+U_{22}-E-i \varepsilon, \\
& L=c \sigma \cdot(D+\nu \omega)+U_{12},
\end{aligned}
$$

and $U_{j k}$ 's are $2 \times 2$ matrices defined by

$$
V=\left(\begin{array}{ll}
U_{11} & U_{12} \\
U_{21} & U_{22}
\end{array}\right) .
$$

Here note that $U_{12}^{*}=U_{21}$. If we set

$$
e^{-i \nu \omega \cdot x} R(E+i \varepsilon) e^{i \nu \omega \cdot x}=\left(\begin{array}{cc}
X & Y \\
Z & W
\end{array}\right)
$$

we have $X, Y, Z, W \in \boldsymbol{B}\left(L^{2}\left(\boldsymbol{R}^{3} ; \boldsymbol{C}^{2}\right), H^{1}\left(\boldsymbol{R}^{3} ; \boldsymbol{C}^{2}\right)\right)$ and

$$
\begin{aligned}
& \left(\begin{array}{cc}
K & L \\
L^{*} & M
\end{array}\right)\left(\begin{array}{cc}
X & Y \\
Z & W
\end{array}\right)=I_{4} \in \boldsymbol{B}\left(L^{2}\left(\boldsymbol{R}^{3} ; \boldsymbol{C}^{2}\right)\right), \\
& \left(\begin{array}{cc}
X & Y \\
Z & W
\end{array}\right)\left(\begin{array}{cc}
K & L \\
L^{*} & M
\end{array}\right)=I_{4} \in \boldsymbol{B}\left(H^{1}\left(\boldsymbol{R}^{3} ; \boldsymbol{C}^{2}\right)\right),
\end{aligned}
$$

that is,

$$
\begin{aligned}
& K X+L Z=I_{2}, \quad L^{*} X+M Z=O \quad \text { in } \boldsymbol{B}\left(L^{2}\left(\boldsymbol{R}^{3} ; \boldsymbol{C}^{2}\right)\right), \\
& K Y+L W=O, \quad L^{*} Y+M W=I_{2} \text { in } \boldsymbol{B}\left(L^{2}\left(\boldsymbol{R}^{3} ; \boldsymbol{C}^{2}\right)\right), \\
& X K+Y L^{*}=I_{2}, \quad X L+Y M=O \text { in } \boldsymbol{B}\left(H^{1}\left(\boldsymbol{R}^{3} ; \boldsymbol{C}^{2}\right)\right), \\
& Z K+W L^{*}=O, \quad Z L+W M=I_{2} \text { in } \boldsymbol{B}\left(H^{1}\left(\boldsymbol{R}^{3} ; \boldsymbol{C}^{2}\right)\right) .
\end{aligned}
$$

Moreover, it follows from $\|R(E+i \varepsilon)\|_{B(\mathscr{F})} \leq 1 / \varepsilon$ that $\|X\|,\|Y\|,\|Z\|_{1},\|W\| \leq$ $1 / \varepsilon$. Since all components of $U_{22}$ and their derivatives are bounded and $\left\|M^{-1}\right\| \leq 1 / \varepsilon$, both $M$ and the inverse $M^{-1}$ belong to $\boldsymbol{B}\left(L^{2}\left(\boldsymbol{R}^{3} ; \boldsymbol{C}^{2}\right)\right) \cap$ $\boldsymbol{B}\left(H^{1}\left(\boldsymbol{R}^{3} ; \boldsymbol{C}^{2}\right)\right)$. Therefore, by the second equality in (4.8) we can see that $(\sigma \cdot D+i) X \in \boldsymbol{B}\left(L^{2}\left(\boldsymbol{R}^{3} ; \boldsymbol{C}^{2}\right), H^{1}\left(\boldsymbol{R}^{3} ; \boldsymbol{C}^{2}\right)\right)$. Since $(\sigma \cdot D+i)^{-1} \in \boldsymbol{B}\left(H^{1}\left(\boldsymbol{R}^{3} ; \boldsymbol{C}^{2}\right)\right.$, 
$H^{2}\left(\boldsymbol{R}^{3} ; \boldsymbol{C}^{2}\right)$ ) follows immediately from $(\sigma \cdot D)^{2}=-\Delta$ (see (4.16) below), we have $X \in \boldsymbol{B}\left(L^{2}\left(\boldsymbol{R}^{3} ; \boldsymbol{C}^{2}\right), H^{2}\left(\boldsymbol{R}^{3} ; \boldsymbol{C}^{2}\right)\right)$. The equalities in (4.8) yield $Z=-M^{-1} L^{*} X$ and $\left(K-L M^{-1} L^{*}\right) X=I_{2} \in \boldsymbol{B}\left(L^{2}\left(\boldsymbol{R}^{3} ; \boldsymbol{C}^{2}\right)\right)$. Moreover, we can also have $X\left(K-L M^{-1} L^{*}\right)$ $=I_{2} \in \boldsymbol{B}\left(H^{2}\left(\boldsymbol{R}^{3} ; \boldsymbol{C}^{2}\right)\right)$ by (4.10), and so, the operator $K-L M^{-1} L^{*}$ with domain $H^{2}\left(\boldsymbol{R}^{3} ; \boldsymbol{C}^{2}\right)$ has a bounded inverse:

$$
X=\left(K-L M^{-1} L^{*}\right)^{-1}, \quad Z=-M^{-1} L^{*}\left(K-L M^{-1} L^{*}\right)^{-1} .
$$

In the same way as above it follows from (4.9) and (4.11) that $M-L^{*} K^{-1} L$ with domain $H^{2}\left(\boldsymbol{R}^{3} ; \boldsymbol{C}^{2}\right)$ has a bounded inverse:

$$
W=\left(M-L^{*} K^{-1} L\right)^{-1}, \quad Y=-K^{-1} L\left(M-L^{*} K^{-1} L\right)^{-1}
$$

Lemma 4.5. Bounded operators $X, Y, Z$ and $W$ have strong limits as $E \rightarrow \pm \infty$ :

$$
\begin{aligned}
s-\lim _{E \rightarrow \pm \infty} X & =(2 L( \pm \omega)-2 i \varepsilon)^{-1} \\
s-\lim _{E \rightarrow \pm \infty} Y & =(2 L( \pm \omega)-2 i \varepsilon)^{-1}( \pm \sigma \cdot \omega), \\
s-\lim _{E \rightarrow \pm \infty} Z & =( \pm \sigma \cdot \omega)(2 L( \pm \omega)-2 i \varepsilon)^{-1}, \\
s-\lim _{E \rightarrow \pm \infty} W & =( \pm \sigma \cdot \omega)(2 L( \pm \omega)-2 i \varepsilon)^{-1}( \pm \sigma \cdot \omega) .
\end{aligned}
$$

Proof of Lemma 4.5. We only give the proof of (4.12) and (4.14) for $E \rightarrow \pm \infty$, because the other cases can be treated similarly. In this proof, the notation $A=B+O_{2}\left(E^{-\ell}\right)$ means that $\left\|(A-B)(-\Delta+1)^{-1}\right\|=O\left(E^{-\ell}\right)$ as $E \rightarrow+\infty$. We write

$$
\begin{aligned}
K-L M^{-1} L^{*}= & -c \sigma \cdot(D+\nu \omega) M^{-1} c \sigma \cdot(D+\nu \omega) \\
& -U_{12} M^{-1} c \sigma \cdot(D+\nu \omega)-c \sigma \cdot(D+\nu \omega) M^{-1} U_{21} \\
& -U_{12} M^{-1} U_{21}+U_{11}+m c^{2}-E-i \varepsilon,
\end{aligned}
$$

and observe that

$$
\begin{aligned}
M^{-1} & =-E^{-1}-\left(U_{22}-m c^{2}-i \varepsilon\right) E^{-2}+O_{2}\left(E^{-3}\right), \\
\nu & =c^{-1} E+O_{2}\left(E^{-1}\right), \quad\left[D, M^{-1}\right]=O_{2}\left(E^{-2}\right) .
\end{aligned}
$$

Now, noting that the Pauli matrices satisfy the following relation:

$$
\sigma_{j} \sigma_{k}+\sigma_{k} \sigma_{j}=2 \delta_{j k} I_{2}, \quad 1 \leq j, k \leq 3,
$$

we obtain 


$$
(\sigma \cdot \omega)(\sigma \cdot D)+(\sigma \cdot D)(\sigma \cdot \omega)=2 \omega \cdot D, \quad(\sigma \cdot \omega)^{2}=1
$$

Thus, it is easily seen that

$$
K-L M^{-1} L^{*}=2 L(\omega)-2 i \varepsilon+O_{2}\left(E^{-1}\right),
$$

and hence,

$$
X-(2 L(\omega)-2 i \varepsilon)^{-1}=O_{2}\left(E^{-1}\right)
$$

since $(-\Delta+1)(2 L(\omega)-2 i \varepsilon)^{-1}(-\Delta+1)^{-1}$ is bounded and $\| X \mid i \leq 1 / \varepsilon$. This implies (4.12), because the operator norm of the left-hand side in (4.18) is uniformly bounded in $E>>1$. Since

$$
\begin{aligned}
& {\left[D_{j}, e^{-i \nu \omega \cdot x}(H-E-i \varepsilon)^{-1} e^{i \nu \omega \cdot x}\right]} \\
& \quad=-e^{-i \nu \omega \cdot x}(H-E-i \varepsilon)^{-1}\left[D_{\jmath}, V\right](H-E-i \varepsilon)^{-1} e^{i \nu \omega \cdot x},
\end{aligned}
$$

it follows that $\sup _{E>>1}\left\|e^{-i \nu \omega \cdot x}(H-E-i \varepsilon)^{-1} e^{i \nu \omega \cdot x}\right\|_{B\left(H^{1}\left(R^{3} ; C^{4}\right)\right)}<+\infty$, and hence

$$
\sup _{E>>1}\|X\|_{B\left(H^{1}\left(R^{3} ; C^{2}\right)\right)}<+\infty
$$

We write

$$
\begin{aligned}
& Z f-(\sigma \cdot \omega)(2 L(\omega)-2 i \varepsilon)^{-1} f \\
& =\left(-M^{-1} L^{*}-(\sigma \cdot \omega)\right) X f+(\sigma \cdot \omega)\left(X-(2 L(\omega)-2 i \varepsilon)^{-1}\right) f
\end{aligned}
$$

The second term in the right-hand side goes to zero by (4.12) for $f \in L^{2}\left(\boldsymbol{R}^{3} ; \boldsymbol{C}^{2}\right)$, and the first term goes to zero by (4.19) if $f \in H^{1}\left(\boldsymbol{R}^{3} ; \boldsymbol{C}^{2}\right)$ since $\left\|-M^{-1} L^{*}-(\sigma \cdot \omega)\right\|_{B\left(H^{1}\left(R^{3} ; C^{2}\right), L^{2}\left(R^{3} ; C^{2}\right)\right)}=O\left(E^{-1}\right)$. On the other hand, the operator norm of $Z-(\sigma \cdot \omega)(2 L(\omega)-2 i \varepsilon)^{-1}$ is uniformly bounded in $E>>1$, and hence (4.14) follows.

Proposition 4.2 immediately follows from Lemma 4.5.

\section{§ 5. Proofs of Theorems 3.1, 3.2 and Proposition 3.3}

Proof of Theorem 3.1 (i). Assumption 2 with $\rho>3$ is supposed. We first note that

$$
\begin{aligned}
& {\left[D^{(\ell)}, \cdots,\left[D^{(1)}, e^{-i \nu(E) \omega \cdot x} Q(E) e^{i \nu(E) \omega \cdot x}\right] \cdots\right]} \\
& \quad=e^{-i \nu(E) \omega \cdot x}\left[D^{(\ell)}, \cdots,\left[D^{(1)}, Q(E)\right] \cdots\right] e^{\imath \nu(E) \omega \cdot x},
\end{aligned}
$$


where each $D^{())}$stands for some $D_{k}, 1 \leq k \leq 3$, and the commutator is the $\ell$-th fold one, $\ell \leq d$. Since $\left[D_{k}, R(E+i 0)\right]=-R(E+i 0)\left(D_{k} V\right) R(E+i 0)$, it follows that

$$
\sup _{|E|>>1}\left\|\left[D^{(\ell)}, \cdots,\left[D^{(1)}, Q(E)\right] \cdots\right]\right\|_{B_{s}}<\infty
$$

for $s \leq \rho / 2$ by Proposition 4.1 and $\rho>3$. Consequently, we hae

$$
\sup _{|E|>>1}\left\|\partial_{x}^{\alpha}\left(e^{-\imath \nu(E) \omega \cdot x} Q_{j k}(E) e^{i \nu(E) \omega \cdot x} 1\right)\right\|_{L_{s}^{2}<\infty}
$$

for each $\alpha$ with $|\alpha| \leq d$ and for $s$ with $3 / 2<s \leq \rho-(3 / 2)$, where $Q(E)=$ $\left(Q_{j k}(E)\right)_{1 \leq j, k \leq 4}$. Since $\theta \neq \omega$, writing $\hat{Q}_{j k}(E, \theta, \omega)$ as

$$
\hat{Q}_{j k}(E, \theta, \omega)=\int e^{-\imath \nu(E)(\theta-\omega) \cdot x}\left(e^{-i \nu(E) \omega \cdot x} Q_{j k}(E) e^{\imath \nu(E) \omega \cdot x} 1\right)(x) d x
$$

(see (2.5)) and integrating by parts, we have the desired result by Proposition 2.1.

Proof of Theorem 3.1 (ii). Assumption 2 with $\rho>3$ is supposed. We set

$$
Q^{ \pm}(\omega)=\left(Q_{j k}^{ \pm}(\omega)\right)_{1 \leq \jmath, k \leq 4}:=-V+V K^{ \pm}(\omega)\left(c \omega \cdot D+V_{\omega}^{ \pm}-i 0\right)^{-1} K^{ \pm}(\omega)^{*} V
$$

Then it follows from our assumption, Corollary 4.4 and (4.7) that $Q^{ \pm}(\omega) \in \boldsymbol{B}_{-s}$ and

$$
s-\lim _{E \rightarrow \pm \infty} e^{-i \nu(E) \omega \cdot x} Q(E) e^{i \nu(E) \omega \cdot x} f=Q^{ \pm}(\omega) f \text { in } L_{s}^{2}\left(\boldsymbol{R}^{3} ; \boldsymbol{C}^{4}\right)
$$

for each $\omega \in S^{2}$ and $f \in L_{-s}^{2}\left(\boldsymbol{R}^{3} ; \boldsymbol{C}^{4}\right)$ if $s \leq \rho / 2$. Thus, by (5.1) we have

$$
\lim _{E \rightarrow \pm \infty} \hat{Q}_{j k}(E, \omega, \omega)=\left(Q^{ \pm}(\omega) \chi_{k}, \chi_{j}\right)_{L^{2}}
$$

Since

$$
\lim _{E \rightarrow \pm \infty}\left(u_{1}^{ \pm}(\nu(E) \omega) u_{2}^{ \pm}(\nu(E) \omega)\right)=K^{ \pm}(\omega)
$$

it follows that

$$
\begin{aligned}
\lim _{E \rightarrow \pm \infty} \frac{f(E, \omega, \omega)}{|E|} & =\left(2 \pi c^{2}\right)^{-1} K^{ \pm}(\omega)^{*}\left(\left(Q^{ \pm}(\omega) \chi_{k}, \chi_{j}\right)_{L^{2}}\right)_{1 \leq \jmath, k \leq 4} K^{ \pm}(\omega) \\
& =\left(2 \pi c^{2}\right)^{-1}\left(\left(\left(K^{ \pm}(\omega)^{*} Q^{ \pm}(\omega) K^{ \pm}(\omega)\right)_{k}, \chi_{j}\right)_{L^{2}}\right)_{1 \leq \jmath, k \leq 4} \\
& =\left(2 \pi c^{2}\right)^{-1}\left(\left(D^{ \pm}(\omega) \tilde{\chi}_{k}, \tilde{\chi}_{\jmath}\right)_{L^{2}\left(R^{3} ; C^{2}\right)}\right)_{1 \leq j, k \leq 2},
\end{aligned}
$$

where $D^{ \pm}(\omega)=\left(D_{j k}^{ \pm}(\omega)\right):=-V_{\omega}^{ \pm}+V_{\omega}^{ \pm}\left(c \omega \cdot D+V_{\omega}^{ \pm}-i 0\right)^{-1} V_{\omega}^{ \pm}$, and $\left\{\tilde{\chi}_{k}\right\}_{k=1,2}$ is the 
canonical basis of $\boldsymbol{C}^{2}$. Writing $x=t \omega+\eta, t \in \boldsymbol{R}, \eta \in \Pi_{\omega}$, we denote by $U^{ \pm}$the multiplication operator with $U_{\omega}^{ \pm}(x):=U_{\omega}^{ \pm}(t, \eta)$. Then by (3.2)

$$
\left(c \omega \cdot D+V_{\omega}^{ \pm}-i 0\right)^{-1}=c^{-1} U^{ \pm}\left(-i \partial_{t}-i 0\right)^{-1}\left(U^{ \pm}\right)^{-1} .
$$

Therefore, we have for each constant vector $u \in C^{2}$ :

$$
\begin{aligned}
V_{\omega}^{ \pm} & \left(c \omega \cdot D+V_{\omega}^{ \pm}-i 0\right)^{-1} V_{\omega}^{ \pm} u(t \omega+\eta) \\
& =i c^{-1} V_{\omega}^{ \pm}(t \omega+\eta) U_{\omega}^{ \pm}(t, \eta) \int_{-\infty}^{t} U_{\omega}^{ \pm}(s, \eta)^{-1} V_{\omega}^{ \pm}(s \omega+\eta) u d s \\
& =V_{\omega}^{ \pm}(t \omega+\eta) U_{\omega}^{ \pm}(t, \eta) \int_{-\infty}^{t} \frac{d}{d s}\left(U_{\omega}^{ \pm}(s, \eta)^{-1}\right) u d s \\
& =V_{\omega}^{ \pm}(t \omega+\eta) U_{\omega}^{ \pm}(t, \eta)\left\{U_{\omega}^{ \pm}(t, \eta)^{-1}-I_{2}\right\} u \\
& =\left(V_{\omega}^{ \pm}(t \omega+\eta)-V_{\omega}^{ \pm}(t \omega+\eta) U_{\omega}^{ \pm}(t, \eta)\right) u,
\end{aligned}
$$

and hence

$$
\begin{aligned}
\left(D^{ \pm}(\omega) u\right)(t, \eta) & =-V_{\omega}^{ \pm}(t \omega+\eta) U_{\omega}^{ \pm}(t, \eta) u \\
& =-c i\left(\partial_{t} U_{\omega}^{ \pm}(t, \eta)\right) u .
\end{aligned}
$$

This yields

$$
\begin{aligned}
\left(D^{ \pm}(\omega) \tilde{\chi}_{k}, \tilde{\chi}_{j}\right)_{L^{2}\left(R^{3} ; c^{2}\right)} & =-c i \int_{\Pi_{\omega}} \int_{R} \partial_{t} U_{\omega}^{ \pm}(t, \eta)_{j k} d t d \eta \\
& =-c i \int_{\Pi_{\omega}}\left(U_{\omega}^{ \pm}(+\infty, \eta)-I_{2}\right)_{j k} d \eta
\end{aligned}
$$

and Theorem 3.1 (i) has been proved.

Proof of Proposition 3.3. In the same way as the proof of Theorem 3.1 (ii), we have

$$
\lim _{E \rightarrow \pm \infty} \frac{f(E, \theta(E), \omega)}{|E|}=\left(2 \pi c^{2}\right)^{-1}\left(\left(D^{ \pm}(\omega) \tilde{\chi}_{k}, e^{\imath \xi \cdot x} \tilde{\chi}_{j}\right)_{L^{2}\left(R^{3} ; c^{2}\right)}\right)_{1 \leq \jmath, k \leq 2},
$$

and so, by using (3.2) and (5.2) we obtain the desired results.

For the proof of Theorem 3.2 the following proposition, may be called the optical theorem, is useful.

Proposition 5.1. Under Assumption 2, we have 


$$
\begin{gathered}
\sigma_{k}(E, \omega)=4 \pi \nu(E)^{-1} \operatorname{Im} f_{k k}(E, \omega, \omega), \quad k=1,2, \\
\sigma_{21}(E, \omega)=-2 \pi \nu(E)^{-1} i\left(f_{21}(E, \omega, \omega)-\overline{f_{12}(E, \omega, \omega)}\right) .
\end{gathered}
$$

for $E \in \Sigma$ and $\omega \in S^{2}$.

Proof. Since the scattering operator $S$ is a unitary operator, the scattering matrices $S(E)$ are unitary operator for a.a. $E \in \Sigma$, and hence

$$
(S(E)-I)^{*}(S(E)-I)=-(S(E)-I)-(S(E)-I)^{*} \text { for a.a. } E \text {. }
$$

Taking account of Proposition 2.1, we get by (5.5)

$$
\begin{aligned}
& \left(\frac{\nu(E)}{2 \pi}\right)^{2} \int_{S^{2}} f\left(E, \theta, \omega^{\prime}\right)^{*} f(E, \theta, \omega) d \theta \\
= & -\frac{\nu(E) i}{2 \pi}\left(f\left(E, \omega^{\prime}, \omega\right)-f\left(E, \omega, \omega^{\prime}\right)^{*}\right),
\end{aligned}
$$

for each $\left(E, \omega, \omega^{\prime}\right)$. By putting $\omega=\omega^{\prime}$, this yields

$$
\begin{gathered}
\left(\frac{\nu(E)}{2 \pi}\right)^{2} \sum_{j=1}^{2} \int_{S^{2}}\left|f_{j k}(E, \theta, \omega)\right|^{2} d \theta=\frac{\nu(E)}{\pi} \operatorname{Im} f_{k k}(E, \omega, \omega), \quad k=1,2, \\
\left(\frac{\nu(E)}{2 \pi}\right)^{2} \sum_{k=1}^{2} \int_{S^{2}} f_{k 1}(E, \theta, \omega) \overline{f_{k 2}(E, \theta, \omega)} d \theta=\frac{i \nu(E)}{2 \pi}\left(-f_{21}(E, \omega, \omega)+\overline{f_{12}(E, \omega, \omega)} .\right.
\end{gathered}
$$

By (3.8) and (3.9), we have completed the proof.

Proof of Theorem 3.2. In the same way as in the proof of Theorem 3.1 (ii), we have

$$
\begin{aligned}
& \lim _{E \rightarrow \pm \infty} \frac{f(E, \omega, \omega)-f(E, \omega, \omega)^{*}}{|E|} \\
& =\left(2 \pi c^{2}\right)^{-1} K^{ \pm}(\omega)^{*}\left(\left(\left(Q^{ \pm}(\omega)-Q^{ \pm}(\omega)^{*}\right) \chi_{k}, \chi_{\jmath}\right)_{L^{2}\left(R^{3} ; c^{4}\right)}\right)_{1 \leq j, k \leq 4} K^{ \pm}(\omega) \\
& =\left(2 \pi c^{2}\right)^{-1}\left(\left(\left(D^{ \pm}(\omega)-D^{ \pm}(\omega)^{*}\right) \tilde{\chi}_{k}, \tilde{\chi}_{j}\right)_{L^{2}\left(R^{3} ; C^{2}\right)}\right)_{1 \leq j, k \leq 2},
\end{aligned}
$$

and

$$
\begin{aligned}
& V_{\omega}^{ \pm}\left(c \omega \cdot D+V_{\omega}^{ \pm}+i 0\right)^{-1} V_{\omega}^{ \pm} \tilde{\chi}_{k}(t \omega+\eta) \\
& \quad=\left(-V_{\omega}^{ \pm}(t \omega+\eta) U_{\omega}^{ \pm}(t, \eta) U_{\omega}^{ \pm}(+\infty, \eta)^{-1}+V_{\omega}^{ \pm}(t \omega+\eta)\right) \tilde{\chi}_{k}(t \omega+\eta) .
\end{aligned}
$$

Since 


$$
\text { il } \begin{aligned}
\left(D^{ \pm}\right. & \left.(\omega)-D^{ \pm}(\omega)^{*}\right) \tilde{\chi}_{k}(t \omega+\eta) \|_{C^{2}} \\
\quad & =\left\|V_{\omega}^{ \pm}(t \omega+\eta) U_{\omega}^{ \pm}(t, \eta)\left(U_{\omega}^{ \pm}(+\infty, \eta)^{-1}-I_{2}\right) \tilde{\chi}_{k}\right\|_{C^{2}} \\
& \leq C(1+|t|+|\eta|)^{-\rho}(1+|\eta|)^{-\rho+1},
\end{aligned}
$$

we obtain by using Fubini's theorem

$$
\begin{aligned}
& \left(\left(D^{ \pm}(\omega)-D^{ \pm}(\omega)^{*}\right) \tilde{\chi}_{k}, \tilde{\chi}_{j}\right)_{L^{2}\left(R^{3} ; C^{2}\right)} \\
& \left.\quad=i c \int_{\Pi_{\omega}}\left(\left(U_{\omega}^{ \pm}(+\infty, \eta)-I_{2}\right)\left(U_{\omega}^{ \pm}(+\infty, \eta)^{-1}-I_{2}\right)\right) \tilde{\chi}_{k}, \tilde{\chi}_{j}\right)_{C^{2}} d \eta \\
& \quad=2 i c \int_{\Pi_{\omega}}\left(\left(1-\operatorname{Re} U_{\omega}^{ \pm}(+\infty, \eta)\right) \tilde{\chi}_{k}, \tilde{\chi}_{j}\right)_{C^{2}} d \eta .
\end{aligned}
$$

Hence, Theorem 3.2 follows from Proposition 5.1.

\section{§ 6. Proof of Proposition 4.1}

We give a sketch of the proof of Proposition 4.1 for only the case $E \geq E_{0}$, because the case $E \leq-E_{0}$ can be treated similarly.

In this section the norm $\|\cdot\|$ stands for $\|\cdot\|_{B_{0}}$ or $\|\cdot\|_{L^{2}\left(R^{3} ; C^{4}\right)}$ for simplicity. By scaling $x \rightarrow E^{-1} x$ we have

$$
\begin{aligned}
& \left\|(1+|x|)^{-s}(H-E-i \varepsilon)^{-1}(1+|x|)^{-s}\right\| \\
& =E^{2 s-1}\left\|(E+|x|)^{-s}\left(H_{E}-1-i \frac{\varepsilon}{E}\right)^{-1}(E+|x|)^{-s}\right\|,
\end{aligned}
$$

where $H_{E}=c \alpha \cdot D+E^{-1}\left(\beta m c^{2}+V(x / E)\right)$. Hence, Proposition 4.1 is equivalent to the following.

Theorem 6.1. There exists $E_{0}>0$ such that for $1 / 2<s \leq 1$ the following estimates are valid:

$$
\left\|(E+|x|)^{-s} R_{E}\left(1+i \frac{\delta}{E}\right)(E+|x|)^{-s}\right\| \leq C_{s} E^{1-2 s},
$$

$\left\|(E+|x|)^{-s}\left(R_{E}\left(1+i \frac{\delta}{E}\right)-R_{E}\left(1+i \frac{\delta^{\prime}}{E}\right)\right)(E+|x|)^{-s}\right\| \leq C_{s} E^{1-2 s}\left|\delta-\delta^{\prime}\right|^{\alpha}$, for any $0<\delta, \delta^{\prime}<1$ and any $E \geq E_{0}$, where $R_{E}(z)=\left(H_{E}-z\right)^{-1}$.

Accepting the following proposition for a while, we give the proof of Theorem 6.1.

Proposition 6.2. There exists $E_{0}>0$ such that for any $s$ with $1 / 2<s \leq 1$ the following estimates are valid. 


$$
\left\|(E+|A|)^{-s} R_{E}\left(1+i \frac{\delta}{E}\right)(E+|A|)^{-s}\right\| \leq C_{s} E^{1-2 s},
$$

$$
\left\|(E+|A|)^{-s}\left(R_{E}\left(1+i \frac{\delta}{E}\right)-R_{E}\left(1+i \frac{\delta^{\prime}}{E}\right)\right)(E+|A|)^{-s}\right\| \leq C_{s} E^{1-2 s}\left|\delta-\delta^{\prime}\right|^{\alpha}
$$

for any $0<\delta, \delta^{\prime}<1$ and any $E \geq E_{0}$, where $A=(1 / 2)(x \cdot D+D \cdot X)$ is the generator of dilation.

Proof of Theorem 6.1. By the resolvent equation $R_{E}(z)=R_{E}(i)+(z-i)$ $R_{E}(z) R_{E}(i)$, we have

$$
R_{E}(1+i \varepsilon)=R_{E}(i)+(1+i \varepsilon-i) R_{E}(i)^{2}+(1+i \varepsilon-i)^{2} R_{E}(i) R_{E}(1+i \varepsilon) R_{E}(i)
$$

Thus, by $\left\|R_{E}(i)\right\|=1$ and $\left\|(E+|x|)^{-s}\right\|=E^{-s}$, the proof of (6.1) is reduced to that of

$$
\left\|(E+|x|)^{-s} R_{E}(i) R_{E}(1+i \varepsilon) R_{E}(i)(E+|x|)^{-s}\right\| \leq C_{s} E^{1-2 s},
$$

for $E \geq E_{0}, 0<\varepsilon<1$. With the aid of (6.3), it suffices for the proof of (6.6) to show that

$$
\sup _{E \geq E_{0}}\left\|(E+|A|)^{s} R_{E}( \pm i)(E+|x|)^{-s}\right\|<\infty,
$$

for each $0 \leq s \leq 1$. The case $s=0$ is obvious, and so the cases $0<s<1$ are reduced to the case $s=1$ by interpolation. Thus, it suffices to show that

$$
\sup _{E \geq E_{0}}\left\|A R_{E}( \pm i)(E+|x|)^{-1}\right\|<\infty .
$$

To do so we write

$$
\begin{aligned}
A R_{E}( \pm i)(E+|x|)^{-1}= & R_{E}( \pm i) A(E+|x|)^{-1} \\
& +R_{E}( \pm i)\left[H_{E}, A\right] R_{E}( \pm i)(E+|x|)^{-1}
\end{aligned}
$$

and

$$
R_{E}( \pm i)=\left(L_{0} \mp i\right)^{-1}-R_{E}( \pm i) V_{E}\left(L_{0} \mp i\right)^{-1}
$$

where $L_{0}=\alpha \cdot D, V_{E}=E^{-1}\left(\beta m c^{2}+V(x / E)\right)$. Since $\left(L_{0}-i\right)^{-1} D$ is bounded and $A=D \cdot x+(3 / 2)$, the operator norm of the first term in the right-hand side of (6.9) is uniformly bounded for $E>>1$, and that of the second term is also uniformly bounded by Assumption 3 for 


$$
i\left[H_{E}, A\right]=H_{E}-V_{E}-x \cdot\left(\nabla V_{E}\right)
$$

(6.8) is now proved and so is (6.1). (6.2) follows from (6.4) in the same way as above. This completes the proof.

It remains to prove Proposition 6.2. Since this proposition can be obtained if we track the proofs in [PSS] with carefully taking account of the $E$ dependence (see [Yaf2], $[\mathrm{J}]$ ), we only give a sketch of the proof. In particular, we omit the proofs of Lemmas 6.3-6.6 below.

Sketch of the proof of Proposition 6.2. We first note that

$$
\sup _{E \geq 1}\left\{\left\|\left[H_{E}, A\right]\left(H_{E}+i\right)^{-1}\right\|+\left\|\left[\left[H_{E}, A\right], A\right]\left(H_{E}+i\right)^{-1}\right\|\right\}<\infty,
$$

by (6.11) and Assumption 3. Fix $f \in C^{\infty}(\boldsymbol{R})$ with

$$
0 \leq f \leq 1, f=0(|x-1| \geq 1 / 2), \quad f=1(|x-1| \leq 1 / 4) .
$$

Then it follows from the definition of $V_{E}$ that

$$
\begin{aligned}
M_{E}^{2}: & =f\left(H_{E}\right) i\left[H_{E}, A\right] f\left(H_{E}\right) \\
& =H_{E} f\left(H_{E}\right)^{2}+f\left(H_{E}\right)\left(-V_{E}-x \cdot\left(\nabla V_{E}\right)\right) f\left(H_{E}\right) \\
& \geq \frac{1}{4} f\left(H_{E}\right)^{2},
\end{aligned}
$$

for any $E \geq E_{0}$ if $E_{0}$ is sufficiently large.

Lemma 6.3. If $\varepsilon \geq 0$ and $\delta>0$, then $1+i \delta$ belongs to the resolvent set of $H_{E}-i \varepsilon M_{E}^{2}$ and

$$
G_{\varepsilon}(\delta):=\left(H_{E}-i \varepsilon M_{E}^{2}-1-i \delta\right)^{-1} \in C([0, \infty) ; \boldsymbol{B}(\mathscr{H})) \cap C^{1}((0, \infty) ; \boldsymbol{B}(\mathscr{H}))
$$

as a function of $\varepsilon$. Moreover, there exists $\varepsilon_{0}>0$ such that $G_{\varepsilon}(\delta)$ satisfies the following estimates.

$$
\begin{gathered}
\left\|f\left(H_{E}\right) G_{\varepsilon}(\delta) \phi\right\| \leq C \varepsilon^{-1 / 2}\left|\left(G_{\varepsilon}(\delta) \phi, \phi\right)\right|^{1 / 2}, \\
\left\|\left(H_{E}+i\right) G_{\varepsilon}(\delta)\right\| \leq C \varepsilon^{-1},\left\|\left(H_{E}+i\right)\left(1-f\left(H_{E}\right)\right) G_{\varepsilon}(\delta)\right\| \leq C,
\end{gathered}
$$

for all $\phi \in \mathscr{H}, E \geq E_{0}, 0<\delta<1$ and $0<\varepsilon<\varepsilon_{0}$ for suitable $\varepsilon_{0}>0$. 
Using this lemma, we can obtain the following.

Lemma 6.4. Define $D_{\varepsilon}:=(E+|A|)^{-s}(\varepsilon|A|+1)^{s-1}, F_{\varepsilon}(\delta):=D_{\varepsilon} G_{\varepsilon}(\delta) D_{\varepsilon}$. Then

$$
\left\|G_{\varepsilon}(\delta) D_{\varepsilon}\right\| \leq C\left(E^{-s}+\varepsilon^{-1 / 2}\left\|F_{\varepsilon}(\delta)\right\|^{1 / 2}\right),
$$

$$
\left\|F_{\varepsilon}(\delta)\right\| \leq C E^{-2 s} \varepsilon^{-1}
$$

for $E \geq E_{0}, 0<\delta<1$ and $0<\varepsilon<\varepsilon_{0}$.

Lemma 6.5. Let $g \in C_{0}^{\infty}(\boldsymbol{R})$. Then

$$
\sup _{E \geq E_{0}}\left\|\left[A, g\left(H_{E}\right)\right]\right\|<\infty .
$$

Combining (6.16), (6.17) and (6.18), we can obtain

\section{Lemma 6.6.}

$$
\left\|\frac{d}{d \varepsilon} F_{\varepsilon}(\delta)\right\| \leq C \varepsilon^{s-1}\left(E^{-s}+\varepsilon^{-1 / 2}\left\|F_{\varepsilon}(\delta)\right\|^{1 / 2}\right)
$$

for $E \geq E_{0}, 0<\delta<1$ and $0<\varepsilon<\varepsilon_{0}$.

Define $K_{\varepsilon}(E, \delta):=E^{2 s-1} F_{\varepsilon / E}(\delta)$. Then by (6.18) and (6.20) we have

$$
\begin{gathered}
\left\|\frac{d}{d \varepsilon} K_{\varepsilon}(E, \delta)\right\| \leq C \varepsilon^{s-1}\left(1+\varepsilon^{-1 / 2}\left\|K_{\varepsilon}(\delta, E)\right\|^{1 / 2}\right), \\
\left\|K_{\varepsilon}(E, \delta)\right\| \leq C \varepsilon^{-1},
\end{gathered}
$$

for $E \geq E_{0}, 0<\delta<1$ and $0<\varepsilon<E_{0} \varepsilon_{0}$. From (6.21) and (6.22) it can be shown that

$$
\sup _{\substack{0<\varepsilon<E_{0} \varepsilon_{0} \\ E \geq E_{0}}}\left\|K_{\varepsilon}(E, \delta)\right\|<\infty,
$$

and that the norm limit $K_{0}(E, \delta):=\lim _{\varepsilon \downarrow 0} K_{\varepsilon}(E, \delta)$ exists in $\boldsymbol{B}(\mathscr{H})$. Therefore, it follows that

$$
\left\|F_{\varepsilon}(\delta)\right\| \leq C E^{1-2 s}, \quad 0<\varepsilon<\varepsilon_{0}, \quad E \geq E_{0}
$$

and that the norm limit $F_{0}(\delta):=\lim _{\varepsilon \downarrow 0} F_{\varepsilon}(\delta)$ exists in $\boldsymbol{B}(\mathscr{H})$. This completes the proof of (6.3). Next we prove (6.4). Taking account of (6.24) and (6.21), we integrate $d K_{\varepsilon}(E, \delta / E) / d \varepsilon$ to have 


$$
\left\|F_{\varepsilon}(\delta / E)-F_{0}(\delta / E)\right\| \leq C \varepsilon^{s-(1 / 2)} E^{(1 / 2)-s}
$$

for $E \geq E_{0}, 0<\delta<1$ and $0<\varepsilon<\varepsilon_{0}$. Since $\left\|D_{\varepsilon} G_{\varepsilon}(\delta)\right\|$ has the same estimate as (6.17), it follows from $(d / d \delta) F_{\varepsilon}(\delta / E)=i E^{-1} D_{\varepsilon} G_{\varepsilon}(\delta / E)^{2} D_{\varepsilon}$ and (6.24) that $\left\|(d / d \delta) F_{\varepsilon}(\delta / E)\right\| \leq C \varepsilon^{-1} E^{-2 s}$, and so

$$
\left\|F_{\varepsilon}(\delta / E)-F_{\varepsilon}\left(\delta^{\prime} / E\right)\right\| \leq C \varepsilon^{-1} E^{-2 s} ; \delta-\delta^{\prime} \mid
$$

for $E \geq E_{0}, 0<\delta, \delta^{\prime}<1$ and $0<\varepsilon<\varepsilon_{0}$. In virtue of (6.25) and (6.26), we get

$$
\begin{aligned}
& \left\|F_{0}(\delta / E)-F_{0}\left(\delta^{\prime} / E\right)\right\| \\
\leq & \left\|F_{0}(\delta / E)-F_{\varepsilon}(\delta / E)\right\|+\left\|F_{\varepsilon}(\delta / E)-F_{\varepsilon}\left(\delta^{\prime} / E\right)\right\|+\left\|F_{\varepsilon}\left(\delta^{\prime} / E\right)-F_{0}\left(\delta^{\prime} / E\right)\right\| \\
\leq & C E^{1-2 s}\left(\varepsilon^{s-(1 / 2)} E^{-(1 / 2)+s}+\varepsilon^{-1} E^{-1}\left|\delta-\delta^{\prime}\right|\right)
\end{aligned}
$$

for $E \geq E_{0}, 0<\delta, \delta^{\prime}<1$ and $0<\varepsilon<\varepsilon_{0}$. Thus, setting $\varepsilon=E^{-1}\left|\delta-\delta^{\prime}\right|^{1-\alpha}$, we obtain (6.4).

\section{Acknowledgement}

I would like to thank Professor T. Ichinose for his valuable advice on Theorem 1.5.

\section{References}

[BGU] Bulla, W., Gesztesy, F. and Unterkofler, K., Holomorphy of the scattering matrix with respect to $c^{-2}$ for Dirac operators and an explicit treatment of relativistic corrections, Comm. Math. Phys., 144 (1992), 391-416.

[BGW] Blancarte, H., Grebert, B. and Weder, R., High- and low-energy estimates for the Dirac equation, J. Math. Phys., 36-3 (1995), 991-1015.

[BH] Balslev, E. and Helffer, B., Limiting absorption principle and resonances for the Dirac operator, Adv. Appl. Math., 13 (1992), 186-215.

[BMP] Boutet de Monvel-Berthier, A., Manda, D. and Purice, R., Limiting absorption principle for the Dirac operator, Ann. Inst. H. Poincaré, A 58 (1993), 413-431.

[BS] Birman, M. S. and Solomjak, M. Z., Spectral Theory of Self-Adjoint Operator in Hilbert Spaces (English translation), Reidel, Dordrecht, 1987.

[CFKS] Cycon, H. L., Froese, R. G., Kirsch, W. and Simon, B., Schrödinger Operators, Springer, Berlin-Heidelberg-New York, 1987.

[F] Faddeev, L. D., Uniqueness of the inverse scattering problem, Vestn. Leningr. Univ., 7 (1956), 126-130.

[J] Jensen, A., High energy resolvent estimates for generalized many-body Schrödinger operators, Publ. RIMS, Kyoto Univ., 25 (1989), 155-167.

[M] Mourre, E., Absence of singular continuous spectrum for certain self-adjoint operators, Comm. Math. Phys., 78 (1981), 391-408.

[N] Newton, R. G., Inverse Schrödinger Scattering in Three Dimensional, Springer, BerlinHeidelberg-New York, 1989.

[PSS] Perry, P., Sigal, I.M. and Simon, B, Spectral analysis of N-body Schrödinger operators, Ann. Math., 114 (1981), 519-567.

[PSU] Pladdy, C., Saitō, Y. and Umeda, T., Resolvent estimates of the Dirac operators, Analysis, 
15 (1995), 123-149.

[S1] Saitō, Y., An asymptotic behavior of the S-matrix and the inverse scattering problem, J. Math. Phys., 25 (1984), 3105-3111.

[S2] - An approximation formula in the inverse scattering problem, J. Math. Phys., 27 (1986), 1145-1153.

[Tha] Thaller, B., The Dirac Equation, Springer, Berlin-Heidelberg-New York, 1992.

[V] Vogelsang, V., Absolutely continuous spectrum of Dirac operators for long-range potentials, J. Funct. Anal., 76 (1988), 67-86

[Yaf1] Yafaef, D. R., Mathematical Scattering Theory: General Theory (English translation), Ame. Math. Soc., Providence, 1992.

[Yaf2] - The eikonal approximation and the asymptotics of the total scattering cross-section for the Schrödinger equation, Ann. Inst. H. Poincaré, A 44 (1986), 397-425.

[Yam1] Yamada, O., On the principle of limiting absorption for the Dirac operator, Publ. RIMS, Kyoto Univ., 8 (1972/73), 557-577.

[Yam2] - A remark on the limiting absorption method for Dirac operators, Proc. Japan Acad., A 69 (1993), 243-246.

[Yam3] — A remark on the limiting absorption method of Dirac operators and Schrödinger operators, preprint. 
